\title{
A Survey on By-Passing Techniques in Wireless Sensor Network
}

\author{
Shazia Almas ${ }^{1}$, Arudra .. ${ }^{2}$ \\ ${ }^{1}$ M. Tech, Department Of Computer Science, Rajiv Gandhi Institute of Technology \& Engineering, Bangalore, India \\ ${ }^{2}$ Assistant Professor, Department Of Computer Science, Rajiv Gandhi Institute of Technology \& Engineering, Bangalore, India
}

\begin{abstract}
In wsn there are numerous assaults, for example, equipment disappointment and programming dirtiness among the various hubs in an exceedingly Wireless detector Network. The detected information from alternative clean locales might likewise get stuck in an exceedingly infected space have to be compelled to no earlier possibility courses of action are created. The arrangement is to By-pass the infected hub progressively utilizing distinctive procedures. After by-passing the bundle from the contaminated territory utilizing BPR system, we are finding the new method for supplying the data from source to destination through the best energy efficient neighbor hub.
\end{abstract}

Keywords: Wireless Sensor Network, Efficient neighbor hub, By-pass system

\section{Introduction}

The detection of certain events in WSN is made viable through data sensing and forwarding from sensor nodes to a control centre or the so-called sink node for further processing. Occurrence of any circumstances normally involves communication of outstanding data to the sink node. However, energy constraints and limitations in other resources restrict direct communications between sensors and the sink node

The sensed data streams indicate the spread of malware attacks, hardware failure and software corruption among the different nodes in a WSN. These factors of node infection can affect generated and incoming data streams resulting in high chances of misleading packet translation and severe communication disruption. This problem is detrimental to applications with real-time measurements that possess stringent QoS requirements. False analyses and wrong decision making will result from infected nodes that produce inaccurate data. The sensed data from other un-infected regions might also get stuck in an infected region.

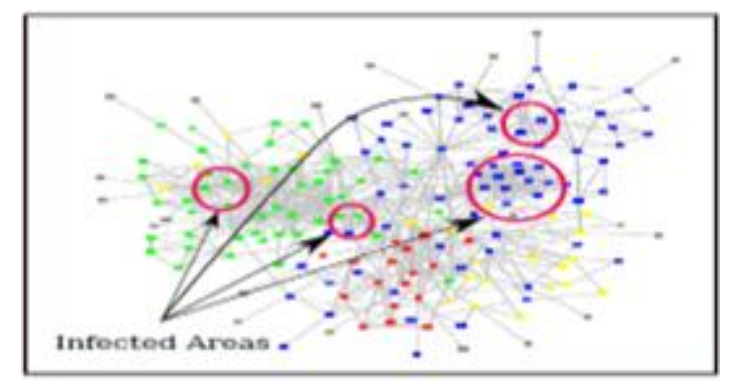

Figure: Nodes Infected as an Infected areas

\section{Related Work}

WSN research community is considered with a few issues including network lifetime[1] which propose Hybrid Multihop routing HYMN algorithm which is a hybrid of two contemporary multi-hop routing algorithm architectures namely Flat multi-hop routing that utilizes efficient transmission distances and hierarchical multi-hop routing algorithm that capitalizes on data aggregation. In [2] detecting anomalies in sensed data in a WSN is essential to identify malfunctioning nodes in order to minimize communication overhead and energy consumption. In [3] sensor localization by distributed angle estimation propose to estimate the angle of departure (AOD) of the emitted waves at each receiving node via frequency measurement of the local received signal strength indication(RSSI) signal. In [4] Wireless Sensor and Actuator networks(WSANs). Using the mobile sink as an example of the actuator to control the movement of a sink has been adopted by researchers in the past to achieve high efficiency in terms of gathering data from the sensors so proposes a novel method based on set packing algorithm and travelling salesman problem. In [5] MANETs have attracted due to mobility and ease of deployment major challenge is to guarantee secure network, certificate revocation is important, issue of certificate revocation to isolate attackers from further participating in network activity. Most of the routing protocols developed for sensor networks employ greedy forwarding algorithm which forwards a packet to a destination node via one hop neighbor[6].It repeats the process until the packet reaches destination and also efficient in reducing

Local minima generally refers to situation in which packet cannot be forwarded to the next hop since no other node has shorter distance to destination. A graph based technique is employed which require the entire network to be stored leading to poor scalability, non graph based technique will result in long routing paths and high energy expenditure. Here holes can be inferred as hot spots caused by traffic congestion. Routing technique to divert traffic away from holes or infected regions and by passing them introduces BOUNDHOLE to discover holes and establish adaptive routes to bypass identified nodes. Another issue is false boundary detection that presents a high risk of falling into loops. To tackle the problem of false boundary GAR is designed which employs a rolling ball method which is attached or hinged at the node having local minima problem and rotate counter clockwise with $\mathrm{R} / 2$ radius and visits unnecessary nodes. 


\section{International Journal of Science and Research (IJSR) \\ ISSN (Online): 2319-7064}

Index Copernicus Value (2013): 6.14 | Impact Factor (2015): 6.391

\section{Objective}

The research is driven by a concern to address the following issues:

- To find a solution that can mitigate the above said problems and get stuck packets out.

- To design a method that can By-Pass the infected areas.

- To minimize the effects of infected nodes

\section{Design Phase}

System Design

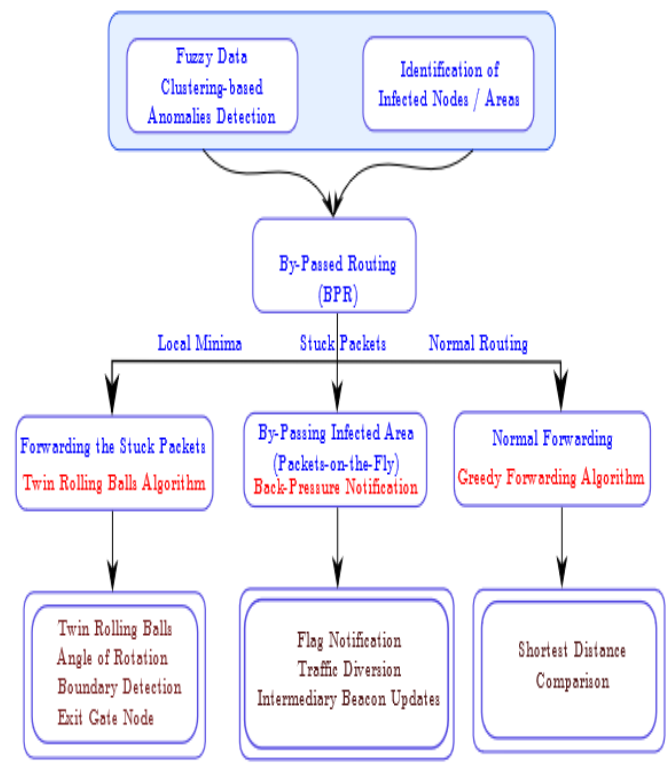

Figure: System Design

\section{Infected/attack region detection}

In this it first finds out the attacked regions or areas through fuzzy data clustering approach where it identifies the attacked data based on the data received. It also finds the data which is attacked through different sensor nodes.

Fuzzy Data Clustering: Infected nodes in communication space identified via Fuzzy data clustering method uses Fuzzy $\mathrm{C}$ algorithm to detect anomalies in sensors. In this algorithm an attempt is made to partition a finite collection of , $\mathrm{n}^{\text {ee }}$ elements $\mathrm{X}=\{\mathrm{X} 1, \mathrm{X} 2, \mathrm{X} 3, \mathrm{X} 4, \ldots . \mathrm{Xn}\}$ into collection of , ${ }^{\text {ce }}$ fuzzy cluster. In a given finite set of data the FCM will return us a set of ${ }^{\mathrm{Ce}}$ cluster centre $\mathrm{C}=\{\mathrm{C} 1, \mathrm{C} 2, \mathrm{C} 3, \ldots \mathrm{Cc}\}$ and also partition matrix $\mathrm{W}=\mathrm{Wij}$ $€[0,1]$ with $i=1,2,3,4 . . j=1$ in which Wij. tells the degree to which elements $\mathrm{Xi}$ belongs to cluster $\mathrm{Cj}$. To determine the shortest route between nodes Dijikstra algorithm is used .In the given network for any provided source node, the shortest route is detected by taking $\mathrm{x}$ and $\mathrm{y}$ coordinate along with threshold value of that node and process continues till the destination is reached.

\section{By-passed routing}

The nodes or regions which is been attacked are by-passed through by-pass routing using different technique. Getting the stucked packets out by using the twin rolling ball algorithm local minima problem,visiting of unnecessary nodes is avoided, detecting false boundary of exit gate node.
To get best path and to reach destination by selecting the shortest path. For better performance and providing efficient energy.

Given a set of sensor nodes $\mathrm{N}=[\mathrm{N} 1, \mathrm{~N} 2, \mathrm{~N} 3, \ldots \mathrm{Nn}]$ on a WSN, a particular node $\mathrm{Ni}$ is considered as infected if it satisfies threshold value based on energy value. If the value is above threshold which is random is considered as infected node. Infected nodes are those which violate normal function of the network so it is detected and by passed When nodes are infected some packets are trapped inside the region and cannot be forwarded to the next hop simply because there is no available node to do so, such that these packets have a high possibility of being dropped if no alternative paths are made to get out of it. So the method called twin rolling ball came into exists. Stuck packets are identified if the node is out of transmission range to send the packet to next node due to local minima problem, hence this concept of twin rolling ball is used.

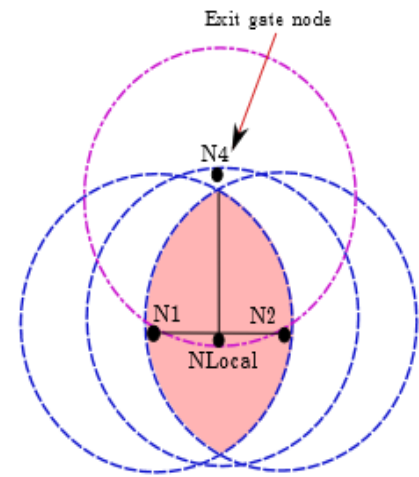

Fig Shows the exit gate node

\section{Conclusion}

In this work, the efficiency of the By-Passed Routing technique where it avoids the infected nodes in network is seen thereby improving the overall performance of network. The infected area comprising of infected nodes called also as anomalous nodes are detected by fuzzy data clustering technique in which first clustering is performed and infected node is calculated based on threshold value in energy model. This information is used by the BPR technique where it finds the alternate path to transfer of packets from source node to destination node with the help of twin rolling balls which define the next forwarding node and reduces the false boundary detection seen in existing rolling ball technique

\section{References}

[1] I. Akyildiz, W. Su, Y. Sankarasubramaniam, and E. Cayirci, "A survey on sensor networks," IEEE Communications Magazine, Vol. 40, No. 8, pp. 102114, 2002.

[2] A. Abdulla, H. Nishiyama, J. Yang, N. Ansari, and N. Kato, "HYMN: A novel hybrid multi-hop routing algorithm to improve the longevity of wsns," IEEE Transactionson Wireless Communications, Vol. 11, no. 7, pp. 2531-2541, July 2012.

[3] N. Arad and Y. Shavitt, "Minimizing recovery state in geographic ad hoc routing," IEEE Transactions on Mobile Computing, Vol. 8, No. 2, pp. 203-217, 2009.

\section{Volume 5 Issue 6, June 2016 www.ijsr.net}




\section{International Journal of Science and Research (IJSR) \\ ISSN (Online): 2319-7064}

Index Copernicus Value (2013): 6.14 | Impact Factor (2015): 6.391

[4] D. Chen and P. K. Varshney, "On-demand geographic forwarding for data delivery in wireless sensor networks," Computer Communications, Vol. 30, No. 1415, pp. $2954-2967,2007$.

[5] M. Ahmadi Livani and M. Abadi, "An energy-efficient anomaly detection approach for wireless sensor networks," Proceedings of 5th International Symposium on Telecommunications, pp. 243248,2010 .

[6] N. Ahmed, S. S. Kanhere, and S. Jha, "The holes problem in wireless sensor networks: a survey," Association for Computing Machinery Special Interest Group on Mobility of systems, Users, Data, and Computing (ACM SIGMOBILE), Review 9, No. 2, pp. 4-18, April. 2005.

[7] Q. Fang, J. Gao, and L. Guibas, "Locating and bypassing holes in sensor networks," Mobile Networks and Applications, Vol. 11, No. 2, pp. 187-200, 2006.

[8] S. Lai and B. Ravindran, "Least-latency routing over timedependent wireless sensor networks," IEEE Transactions on Computers, Vol. 62, No. 5, pp. 969983, 2013.

[9] W. Liu, H. Nishiyama, N. Ansari, J. Yang, and N. Kato, "Cluster-based certificate revocation with vindication capability for mobile ad hoc networks," IEEE Transaction on Parallel Distributed System, Vol. 24, No. 2, pp. 239-249, Feb. 2013.

[10] S. Chen, G. Fan, and J. hong Cui, "Avoid 'void' in geographic routing for data aggregation in sensor networks," International Journal of Ad Hoc and 2016.

[11] Ashwini and A. S., "Information dissemination between nodes of different intersections intersection in city environment using hop greedy routing protocol (BAHG)," Int. J. Ethics Eng. Manag. Educ., vol. 1, no. 4, pp. 232-236, Apr. 2014

[12] S. Subramanian, S. Shakkottai, and P. Gupta, "On optimal geographic routing in wireless networks with holes and non-uniform traffic," in Proc. IEEE 26th Int. Conf. Comput. Commun., May 2007, pp. 1019-1027

[13] H. Nakayama, N. Ansari, A. Jamalipour, and N. Kato, "Faultresilient sensing in wireless sensor networks," Comput. Commun., vol. 30, no. 11-12, pp. 2375-2384, Sep. 2007.

Volume 5 Issue 6, June 2016 www.ijsr.net 